\title{
10. Der Verleger Ernst Rohmer
}

m 12. Juli 1911 übersandte Oskar
Beck seinem Verlegerkollegen
Georg Hirth einen Geburtstagsgruß. Der Mitbesitzer der führenden libe-
[...] die tief bewegte Zeit, in der unser teures Vaterland nach Gestaltung rang. Ernst Rohmer an Friedrich Theodor Vischer, 28. Juli 18871

ralen Zeitung in Süddeutschland, der 184,8 ins Leben gerufenen «Münchner Neuesten Nachrichten», feierte am Folgetag seinen 7o. Geburtstag. Es sei 4,2 Jahre her, so schrieb Oskar Beck, dass er aus dem Munde seines längst verstorbenen Stiefvaters Ernst Rohmer, «eines politischen Freundes Julius Knorrs und Mitbegründers der bayerischen Fortschrittspartei», Hirths Namen zum ersten Mal vernommen habe. «Lebhafte Hoffnungen für die nationale und liberale Sache in München und Bayern» habe die Männer verbunden. ${ }^{2}$

\section{Der liberale Patriot Ernst Rohmer}

Durch Ernst Rohmer wurde C.H.Beck in Nördlingen zu einem Verlag des politischen Liberalismus in Bayern. Schon Friedrich Schulze bemerkte, dass «die Agitationsliteratur» der 4,8er Revolution «sehr schnell und man kann sogar sagen: zu schnell verschwand», aber sie habe «selbst in der politischen Wissenschaft [...] ihre sichtbaren Spuren»zurückgelassen, "wie sich aus der Verlagstätigkeit von C.H.Beck ganz gut ersehen lässt». ${ }^{3}$ Die Autoren, die das Verlagsprogramm nach der Jahrhundertmitte profilierten, waren gestandene Liberale. Sie alle trieb die Frage um, wie der Verfassungsstaat auszusehen habe, der liberale und nationale Ideen zusammenführen und Deutschlands Einheit garantieren könne. Nach der gescheiterten Revolution von 1848 wurden sie in den Bann der «Realpolitik» gezogen. Die Herstellung der nationalen Einheit hatte Vorrang vor dem demokratischen Ausbau Deutschlands. In dem lange abgelehnten Deutschen Bund sah man ein probates Mittel, die staatliche Einheit zu schaffen.

Rohmer lieh als Verleger den Forderungen der Kleindeutschen Partei in Bayern seine Stimme. Im schwelenden Konflikt zwischen Preußen und 

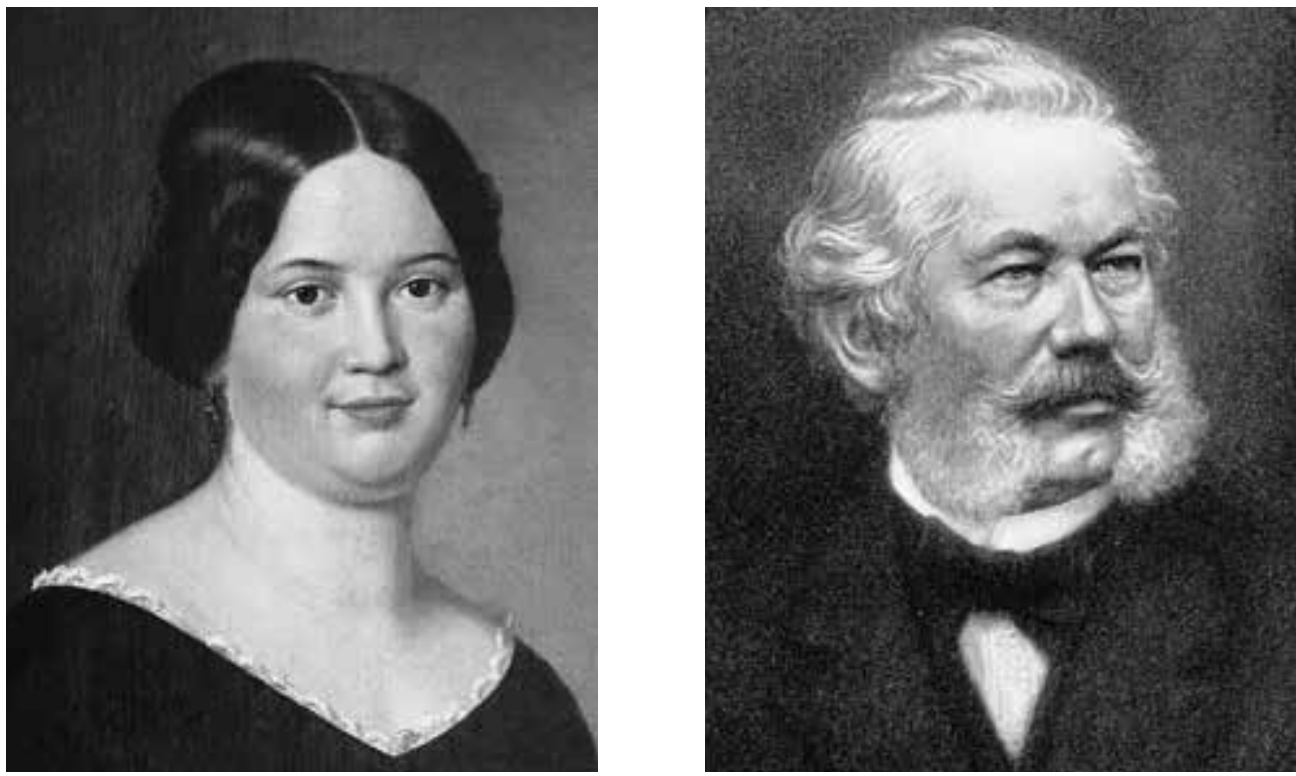

Eugenie Rohmer, die Witwe des Verlegers Carl Beck, und ihr zweiter Ehemann Ernst Rohmer

Dänemark um die Herzogtümer Schleswig und Holstein unterstützte er mit seinen «Fliegenden Blättern» die borussische Seite. ${ }^{4}$ Er kämpfte gegen das «in Süddeutschland immer noch starke Vorurteil gegen Preußen». ${ }^{5}$ Die deutsche Einigung sollte unabhängig von der Haltung Österreichs vollzogen werden. Folgerichtig trat er 1859 als einer der ersten Bayern dem neu gegründeten Deutschen Nationalverein bei. Der liberale Schub der «Neuen Ära» veranlasste ihn in diesem Jahr zu der Bemerkung, dass man publizistisch die «Sympathien nur für ein energisch-liberales Preußen» äußern dürfe, nicht für «Preußen quand même». ${ }^{6}$ Aber er lief nicht, wie mancher andere, der durch die Reformen in Österreich begeistert war, in das großdeutsche Lager über. Auch die Parlamentarisierung des Regierungssystems war für Rohmer nicht verhandelbar. Anfang 1863 kamen in Nördlingen die intellektuellen und politischen Köpfe der kleindeutsch orientierten liberalen Bewegung zusammen, darunter Karl Brater, die Rechtsanwälte Joseph Völk und Marquard Barth, der Erlanger Theologe Johann Christian Konrad Hofmann und sein Kollege Heinrich Marquardsen von der Juristischen Fakultät und der Weingutsbesitzer Ludwig Andreas Jordan aus dem pfälzischen Deidesheim. ${ }^{7}$ Ernst Rohmer hatte sie in sein Haus geladen, und in seinen vier Wänden wurde die bayerische Fortschrittspartei gegründet, die bei den Landtagswahlen im selben Jahr 16 Mandate erlangte. 
Im Deutschen Krieg von 1866 stand er auf der Seite Preußens und polemisierte gegen «die fanatische Kriegslust» der «Demokraten und Großdeutschen».8 Kurzzeitig fürchtete er den Sieg Österreichs. Damals fand allerdings auch Bismarcks Politik keine uneingeschränkte Zustimmung, denn «die jetzige Lage» sei «doch wesentlich durch den Wahn Bismarck's, die Regeneration Deutschland's ohne das Volk und die Freiheit unternehmen zu können», herbeigeführt, «eine Verwegenheit, die sich sicher strafen dürfte [...]. Und dann die lügenhafte Verquickung mit der protestantischen Pfäfferei! Was sind das für Proklamationen und für Tagesbefehle! Wenn man für Preußen seit Jahren eingestanden ist, so weiß man jetzt kaum mehr, was man sagen soll, denn auf die großen prinzipiellen Entwicklungen verstehen sich die Waffen nicht.» ${ }^{9}$ Umso größer war die Erleichterung, als der Sieg Preußens feststand: «Die Misere der Kleinstaaterei» konnte überwunden werden und «der Deutsche ein Vaterland» bekommen. ${ }^{10}$ Rohmer bekannte sich zu Bismarcks Realpolitik, um die deutsche Frage zu lösen. 1867 agitierte er in einer kleinen Schrift «Unsere Lage und unsere Pflicht» für den Anschluss Bayerns an den Norddeutschen Bund. An Heinrich von Treitschke schrieb er unmittelbar vor der konstituierenden Sitzung des Norddeutschen Reichstags, in München gebe es immer noch zu viele Partikularisten in und außerhalb der Regierung. Doch habe Wilhelm I. in seiner «so wundervollen und patriotischen Thronrede» einen guten Anfang gemacht, und so wolle man hoffen, «dass von den Alpen bis zum Meere der Eine deutsche Staat recht bald begründet wird!»11 Die preußisch-süddeutschen Bündnisse begrüßte er uneingeschränkt. 1868 kandidierte er bei den Wahlen zum Zollparlament für die bayerische Fortschrittspartei. Der Erfolg blieb ihm verwehrt. Im folgenden Jahr gründete er mit liberalen Gesinnungsgenossen einen «politischen Bürgerverein» in Nördlingen, der alle seine «Zusammenkünfte pflichtgemäß dem Magistrat als der zuständigen Überwachungsbehörde» anzeigte. ${ }^{12}$

C.H.Beck in Nördlingen richtete unter Rohmer sein Programm konsequent gegen den bayerischen «Ultramontanismus» aus, der, wie Karl Brater schrieb, «in München die Basis seiner kirchlich-politischen und wissenschaftlichen Operationen» hatte. ${ }^{13}$ Die 1857 in Nördlingen herausgegebenen «Fliegenden Blätter» waren nicht zuletzt ein Versuch, dem Eindruck entgegenzutreten, dass allein die «ultramontanen», sprich katholischen Blätter, «den Begriff der freien Presse in Bayern vor Extinktivverjährung und gänzlicher Vergessenheit» bewahrten. Die «Begrenzung der Wissenschaft durch den Glauben» wurde kategorisch abgelehnt. ${ }^{14}$ Der Verlag erreichte so das liberale Besitz- und Bildungsbürgertum vor allem in den 
neubayerischen protestantischen Gebieten in Ober- und Mittelfranken, in weiten Teilen Schwabens und in den größeren Städten. Seine Schriften verkaufte der Verlag auch an der Haustür und wurde deshalb mit der einen oder anderen Beschwerde katholischer Buchhändler abgestraft. ${ }^{15}$

Die heftige Abneigung gegen den Ultramontanismus mündete nicht in eine undifferenzierte antikatholische Polemik. Im Gegenteil. Rohmer öffnete seinen Verlag katholischen Wissenschaftlern, die die Antimodernisten in den eigenen Reihen kritisierten. So druckte C.H.Beck die Schrift «Die christliche Gesellschaftsordnung und die neue Zeit» des heute weitgehend vergessenen Bamberger Kirchenhistorikers Johann Spörlein, weil Rohmer «diese Art Apologetik» durchaus «geistvoll, liberal und in ihrer Art originell» erschien, «um so origineller, als sie von einem katholischen Gelehrten kommt». Spörlein schrieb an Rohmer, seine «Betrachtung» sei «gegen jene traurigen Leute gerichtet, welche alle Erstrebungen und Errungenschaften der Neuzeit - den Fortschritt der staatlichen und persönlichen Freiheit, die Religionsfreiheit, die Ergebnisse der Naturwissenschaft [...] als etwas Gefährliches und Böses ausgeben». Er versuche darzustellen, «dass gerade das mittelalterliche Leben, für welches sie schwärmen, mit heidnischen Bestandteilen überall vermengt war, dagegen die neue Zeit die christlichen Gedanken in reineren - allerdings neuen - Formen zu verwirklichen bestrebt ist». Rohmer war angetan, wie er an Bluntschli schrieb: «Mir scheint die Schrift so recht verwandt mit unserm Ideenkreise, und wenn auch vielleicht einzelne Irrtümer mit unterlaufen, so scheint mir doch der aufgerollte Abriss schlagend und auch für das ehrliche orthodoxe Publikum aufhellend. Sie trifft wie mir scheint katholische und protestantische Pfaffen gleicherweise.» ${ }^{16}$

«Schranken gegen Priester-Willkür» sollten nicht nur durch politische Agitation, sondern auch durch das geschriebene Wort errichtet werden. ${ }^{17}$ Ernst Rohmer unterstützte Bismarcks Kulturkampf. Die Auseinandersetzungen um die Unfehlbarkeit des Papstes riefen den Nördlinger Verleger auf den Plan. Dem Machtanspruch des Vatikans sollte ein Riegel vorgeschoben werden. Den Auftakt machte das «Sendschreiben an einen deutschen Bischof des vatikanischen Concils», das der liberale britische Katholik Lord Acton verfasst hatte. Dann öffnete Rohmer seinen Verlag den katholischen Kirchenhistorikern Ignaz von Döllinger und Johann Friedrich, als diese gegen das Infallibilitätsdogma des Papstes protestierten, sich vom Ersten Vaticanum distanzierten und den Grundstein der Altkatholischen Kirche legten. In der ersten Hälfte der 1870er Jahre erschienen in dem protestantischen Verlag Quelleneditionen der katholischen Gelehrten, 
die mit der historisch-kritischen Methode die Überlegenheit eines nicht ultramontanen Katholizismus demonstrieren wollten. «In dem Augenblicke, wo die katholische Kirche vor allem, dann aber auch andere kirchliche Richtungen die Konsequenzen ihrer Dogmen bis zum äußersten Extrem führen, bis zum Unsinn, muss die Zeit der Umkehr nahe sein», ließ Ernst Rohmer den Theologen wissen. ${ }^{18}$ Allerdings wies Rohmer schon zu Beginn der Verhandlungen darauf hin, dass «Quellenschriften für den Verleger eine gefährliche Sache» seien, selten etwas abwürfen und dass deshalb kein Honorar vergütet werden könnte. ${ }^{19}$ Mit diesen Schriften erreichte C.H.Beck den Teil des katholischen Bürgertums, der den liberalen Staat und die aufgeklärte Wissenschaft nicht ablehnte. Die Mehrzahl der Publikationen Döllingers, die geplant waren und für die bereits Verträge abgeschlossen waren, erschienen erst zwei Dezennien später, als der Verlag bereits von Ernst Rohmers Nachfolger, Oskar Beck, geführt wurde. ${ }^{20}$ Denn der katholische Theologe ließ sich viel Zeit, seine Manuskripte abzuschließen, und musste immer wieder an seine vertraglichen Verpflichtungen erinnert werden. Die «Geschichte der jesuitischen Moral», für die Döllinger schon Anfang 1873 einen Vertrag unterzeichnete, konnte erst 1889 der Öffentlichkeit übergeben werden. ${ }^{21}$

Den Deutsch-Französischen Krieg begrüßte Ernst Rohmer ohne Einschränkung. «Für Bayern war das Jahr 1870 eine wahre Erlösung», rief er rückblickend aus. ${ }^{22}$ An Bluntschli schrieb er am 22. Juli 1870, drei Tage nach der französischen Kriegserklärung: «Welche Gnade, dass wir das erleben durften.» ${ }^{23}$ Die Proklamation des Deutschen Kaiserreichs im Spiegelsaal von Versailles war eine «wahre Erlösung». Die verfassungspolitischen Ziele waren erreicht. Der «Aufbau eines neuen herrlicheren deutschen Reiches als es je gewesen» konnte beginnen. ${ }^{24}$ Offen bekannte Rohmer sich jetzt zu Bismarcks Politik und kritisierte dessen Gegner, wie den Heidelberger Historiker Georg Gottfried Gervinus. ${ }^{25} 1871$ ließ er sich als Kandidat der Fortschrittspartei für den Reichstag aufstellen. Doch auch diesmal wurde er nicht gewählt: Die Wahl verlor der freisinnige Rohmer im ländlichen Raum; er unterlag, bei insgesamt 15 124, abgegebenen Stimmen, dem Kandidaten des Zentrums mit 358 Stimmen. Das «Nördlinger Anzeigeblatt» vom 8. März klagte, dass es eine «Sisyphus-Arbeit» sei, «gegen den Wahn der durch die Einflüsterungen des Klerus blind sich leiten lassenden ungeheuren Mehrheit» zu kämpfen. ${ }^{26}$ Die wenig später erfolgte Gründung eines katholischen Volksblatts und einer katholischen Buchhandlung in Nördlingen kommentierte Rohmer entsprechend bitter. Ein «leidenschaftlicher und jesuitischer katholischer Stadtpfarrer» habe in 
kurzer Zeit bei den Bauern im Ries zo ooo Gulden aufgetrieben «und allein dafür ausgegeben, ein Beweis, welche Macht diese Fanatiker auf das Landvolk üben. Die Konkurrenz ist mir natürlich ganz gleichgültig, aber die Aussicht, dass der konfessionelle Friede in Stadt und Land gründlich gestört werden wird und dass ich bei meinen vielen Geschäften genötigt werden soll, mich mit dem ultramontanen Pack in meinem Blatte herumzuschlagen, ist bitter.» ${ }^{27}$

1874, scheiterte Rohmer erneut mit seiner Kandidatur für den Reichstag im schwäbischen Wahlkreis Donauwörth, Neuburg und Nördlingen. Er habe sich «gewissermaßen als Opfer der Partei für die Reichstagswahlen hergeben müssen», gestand er Bluntschli. Er habe mehrmals abgelehnt, endlich jedoch angenommen, nachdem er sich überzeugen konnte, dass seine Kandidatur «allein im Stande wäre, wenigstens eine starke Minorität $\mathrm{zu}$ erzielen». ${ }^{28}$ Erneut scheiterte er an dem «wahrhaft drückenden Aufmarsch des schwarzen Landsturmes». ${ }^{29}$ Auf Reichsebene konnte er den parteipolitischen Liberalismus nicht vertreten, doch als Repräsentant des Wirtschaftsbürgertums beeinflusste er die städtische Politik: Er saß drei Jahrzehnte lang im Magistratsrat von Nördlingen und gründete Anfang der 1870 er Jahre nach dem Vorbild von Hermann Schulze-Delitzsch eine genossenschaftlich organisierte Darlehensbank mit Solidarhaftung, deren Geschäfte er bis in sein letztes Lebensjahr führte. ${ }^{30}$ Und er machte nach der Reichsgründung als erster Vorsitzender des süddeutschen Buchhändlervereins im Börsenverein der deutschen Buchhändler und im bayerischen Buchhändlerverein einige Jahre Standespolitik, wo er gegen die «Schleuderei» der Bücher und für die Einführung eines festen Ladenpreises Stellung bezog. ${ }^{31}$

Die Diskussionen um die soziale Frage und den Aufstieg der Sozialdemokratie verfolgte der Nördlinger Unternehmer aufmerksam. Seines Erachtens legten die Liberalen zu wenig Gewicht auf die «Arbeiterfrage». Er plädierte für die Einführung des allgemeinen und direkten Wahlrechts, da «nur dieser Wahlmodus dem Arbeiterstand die Möglichkeit einer Vertretung» gewähre. Nur so könne «falschen Propheten» wie dem linkshegelianischen Intellektuellen Ferdinand Lassalle Einhalt geboten werden. ${ }^{32}$ Den Triumph der radikalen «Demokraten» und der Linken fürchtete Rohmer wie andere Repräsentanten des liberalen Wirtschaftsbürgertums nicht weniger als den Vormarsch der Ultramontanen. 1878, mitten in den Diskussionen um das Sozialistengesetz, griff Ernst Rohmer auf Positionen seines Bruders Friedrich aus dem Jahr 184,8 zurück und forderte, «die berechtigten Interessen des vierten Standes zur Geltung zu bringen». Die Regierung 
dürfe nicht nur repressiv, sondern müsse reformatorisch handeln, sonst sei «eine schwere Revolution unausbleiblich». Angesichts des rasanten Wachstums der Sozialdemokratie falle der Monarchie die «Hauptaufgabe» $\mathrm{zu}$, «denn dass liberale und konservative Geldsäcke ohne Zwang von oben barmherzig werden und ihre Schuldigkeit von selbst tun, ist noch nicht erlebt worden!»35 Der Aufstieg der Industriellen wurde mithin nicht nur als Herausforderung bürgerlicher Werte, sondern als Gefahr des sozialen Friedens erachtet. Nachdem der russische Zar Alexander II. 1881 einem Attentat zum Opfer gefallen war, sah Rohmer «den Beginn großer revolutionärer Bewegungen für die slawischen Völker unter Russlands Szepter» voraus. Im Deutschen Reich erschienen ihm Bismarcks «Ideen» für die Arbeiterschaft «groß und ausführungswürdig»; allein die Form, «unter der er sie anstrebt», seien «doch recht bedenklich und zweifelhaft, namentlich die Centralisation in den Händen des Staates». ${ }^{34}$

Der Verleger verwirklichte in seinem Sortiment das Programm der Fortschrittspartei, die eine konstitutionelle Monarchie forderte, in der «die Verfassung das unlösbare Band» sein sollte, «welches Fürst und Volk zusammenhält». Konservative Positionen waren erlaubt, sozialistische Vorstellungen hingegen verpönt. «Eine feste liberale Regierung» sollte die Rechte der Bürger achten. Rohmer nutzte seinen Verlag, um dafür zu werben, dass in der Gesetzgebung «die strenge und konsequente Verwirklichung des verfassungsmäßigen Rechtsstaates die erste und unbedingte Notwendigkeit» sei. ${ }^{55}$ Deshalb baute er C.H.Beck energisch zu einem juristischen Verlag aus, der die Justiz- und Verwaltungsreformen, die nach 1848 in Bayern durchgeführt wurden, begleitete. Die 1857 in den «Fliegenden Blättern» postulierte Trennung der Verwaltung von der Justiz wurde in Bayern 1862 umgesetzt. ${ }^{36}$ Die juristische und bürokratische Umsetzung dieser zentralen Forderungen des politischen Liberalismus brauchte nicht nur einen neuen Beamtenapparat, sondern auch neue Fachorgane und Publikationsreihen. Die legislativen Akte mussten der Öffentlichkeit durch Textausgaben und Kommentare kommuniziert werden. Im Verlagskatalog von 1863, der das 100-jährige Bestehen der Firma feierte, übertraf die Zahl der juristischen Publikationen knapp die der theologischen Titel. Das Strafgesetzbuch von 1861 wurde ebenso kommentiert wie die Gerichtsordnung, die Wehrverfassung ebenso wie das Heimat- und Armengesetz und die Zivilprozessordnung. Ab 1864, erschien die «Bayerische Notariatszeitung», die mit der Einführung des Notariats einherging. 


\section{Der Verlag nach der Reichsgründung}

Die Reichsgründung von 1871 stellte den Verlag vor eine neue Herausforderung: Hatte er sich auf dem Gebiet des bayerischen Rechts eine führende Position erarbeitet, so musste er mit dem Eintritt des Königreichs Bayern in das Deutsche Reich seine Kompetenz auf dem Gebiet des Reichsrechts unter Beweis stellen. ${ }^{37}$ Ernst Rohmer erkannte diese Notwendigkeit sofort und gewann den bayerischen Juristen Emil Riedel, der noch im selben Jahr die Reichsverfassung und die wichtigsten Administrativgesetze des Deutschen Reiches kommentierte. Das Buch des späteren Finanzministers übersandte er Heinrich von Treitschke. Der damals noch nicht 40-jährige Verfasser, so ließ er den Historiker wissen, sei einer der «tüchtigsten jüngeren Juristen», der sich «seit mehreren Jahren in München als einer der entschiedensten und tüchtigsten Vorkämpfer für die deutsche Sache bewährt» habe. Sein Werk über die «Reichsverfassungsurkunde vom 16. April 1871", das eine systematische Darstellung der Grundzüge des deutschen Verfassungsrechts enthalte, sei «voraussichtlich» der erste Versuch, «eine Art Grundriss» des Reichsverfassungsrechts aufzustellen. Er bat Treitschke, sich dafür einzusetzen, dass die Redaktion der von ihm mit herausgegebenen «Preußischen Jahrbücher» «geneigt ist, darauf aufmerksam zu machen». 38

Rohmer setzte nicht nur auf Praktiker als Autoren, sondern auch auf junge Juristen, die bisweilen noch Regierungsassessoren waren, wenn sie ihren Verlagsvertrag unterschrieben. Aus vergleichsweise bescheidenen Anfängen entstanden dann große Kommentare und Editionen, die richtungweisend waren und blieben. Eng gewebte Netzwerke in die Verwaltung und Rechtsprechung, aber auch zu liberalen Politikern wie Karl Brater halfen bei der Rekrutierung neuer Autoren. ${ }^{39}$ Mit jedem erfolgreichen juristischen Projekt wuchs das Renommee des Verlags, der somit zumindest für bayerische Juristen zur ersten Adresse wurde. Doch Ernst Rohmer schaffte den Sprung auch über Bayern hinaus und war gleichermaßen erfolgreich als Verlag des bayerischen Landesrechts wie des deutschen Reichsrechts. 1883 erschien das älteste, auch heute noch als Loseblattsammlung aktualisierte juristische Fachbuch Deutschlands bei C.H.Beck, der von Robert von Landmann verfasste «Kommentar zur Gewerbeordnung für das Deutsche Reich». ${ }^{40}$ «Jedes bedeutsamere bayerische Gesetz und Reichsgesetz» wurde in einer selbständigen Textausgabe herausgebracht; ${ }^{41}$ diese Gesetzesausgaben wurden, so erforderlich, überarbeitet und regelmäßig kommentiert. Die von Karl Brater verantwortete, bereits 
erwähnte Edition der bayerischen Verfassungsurkunde etwa erschien 1872 in der Bearbeitung von Georg Pfeil in vierter Auflage. Lehrbücher für den akademischen Unterricht kamen hinzu. Markenzeichen der juristischen Texte, die bei C.H.Beck erschienen, waren landesweit ihre roten Einbände.

Stillstand war zu vermeiden, auch auf dem Zeitungsmarkt. Unter Ernst Rohmers Leitung nahm das «Nördlinger Wochenblatt», das seit 1.April 1863 als «Nördlinger Anzeigeblatt» dreimal wöchentlich erschien, wieder den Charakter einer politischen Zeitung an. 1871 kam das Blatt dann viermal in der Woche heraus, Montag, Mittwoch, Freitag und Sonnabend. 1887 erschien die Zeitung bei vergrößertem Format dann täglich. ${ }^{42}$

Ernst Rohmer öffnete seinen Verlag den Freunden, die sich in Zürich um Theodor und Friedrich Rohmer geschart hatten. Zu dem Kreis gehörten die Schweizer Johann Caspar Bluntschli, Heinrich von Orelli (1783-186o), Heinrich Schultheß (1815-1885) und Heinrich Hottinger (1826-1876) sowie die Deutschen Gustav Widenmann (1812-1876), Adolf Widmann (18181878), Friedrich M. Duttenhofer (1810-1859) und Alexander Bruckmann (1806-1852)..$^{43}$ In Nördlingen kam Karl Brater hinzu. Johann Caspar Bluntschli wurde zum Starautor, Heinrich Schultheß gab den Geschichtskalender heraus, Heinrich Hottinger arbeitete an der Edition der Schriften von Friedrich Rohmer mit, Gustav Widenmann veröffentlichte 1859 aus dem Nachlass Theodor Rohmers Schrift «Die Religion Jesu» und schrieb über «Die Religion und das Recht der Welt» (1852), «Politik und Kirchentum des Abendlandes» (1855) sowie die «Bundesreform» (1861), und der Ludwigsburger Tierarzt Friedrich M. Duttenhofer - der Surinam bereist hatte, um zu klären, ob die niederländische Kolonie für europäische Auswanderer attraktiv sei - handelte 1855 «Über die Emanzipation der Neger. Ein Versuch zur Aufstellung humaner Prinzipien» und untersuchte drei Jahre später «Die acht Sinne des Menschen», die er «nach ihren körperlichen und geistigen Beziehungen für denkende Leser jeder Art in Briefen» darstellte. Die Freunde aus dem Bluntschli-Rohmer-Kreis und ihre Verwandten unterstützte Rohmer auch materiell, wenn sie wie Karl Brater oder Heinrich Schultheß in Not gerieten. ${ }^{44}$

Als Verleger ließ sich Ernst Rohmer nicht nur das Andenken seiner Brüder Friedrich und Theodor angelegen sein. Er überzeugte auch entferntere Verwandte, für ihn zu schreiben, wenn er sich von einer Veröffentlichung Gewinn versprach. «Hebammendienste» wollte er leisten, wie er es formulierte. Seinen Vetter Karl Christian Planck überredete er im Mai 1872, «eine ernste gedankenreiche Schrift über den Darwinismus» zu verfassen. Planck war Gymnasiallehrer in Blaubeuren und gerade dabei, ein umfas- 
sendes philosophisches System zu entwickeln, das so komplex war, dass nur er selbst es verstand. ${ }^{45}$ Mit Hilfe des Verwandten wollte Rohmer an der zeitgenössischen Diskussion über Darwins Lehre partizipieren. Er bat Planck, seine «Ideen über diesen Gegenstand in eine elegante für einen großen Kreis verständliche Form» zu bringen. Der schreibfreudige Planck zögerte nicht lange und brachte noch im selben Jahr 200 Seiten über «Wahrheit und Flachheit des Darwinismus» zu Papier. Dieser «Denkstein zur Geschichte heutiger deutscher Wissenschaft» wurde in 850 Exemplaren gedruckt. Auch wenn einzelne Theologen und Philosophen in der Auseinandersetzung mit Darwins Evolutionslehre auf Planck rekurrierten, wurde der Band keineswegs «ein bedeutender Erfolg», wie Rohmer gehofft hatte. Schon Rezensenten zu finden war ein schwieriges Unterfangen; dafür war der Stil zu ungelenk und die Sprache zu dunkel. Rohmer selbst bemerkte: «Ich konnte nur blättern in der stattlichen Schrift und erstaunte über Dein umfassendes Wissen. Nur fürchte ich, dass ich von letzterem zu wenig besitze, um Deinen Ideengang ins Einzelne verfolgen zu können.» Aber er hatte so kalkuliert, dass sein finanzieller Verlust nicht allzu groß war. Ein Honorar erhielt der Verfasser nicht, weil zu wenige Exemplare verkauft wurden. ${ }^{46}$ Fünf Jahre später veröffentlichte Rohmer dennoch Plancks Studie «Logisches Kausalgesetz und natürliche Zwecktätigkeit. Zur Kritik aller Kantischen und nachkantischen Begriffsverkehrung», obwohl abzusehen war, dass das Buch ebenfalls nicht gehen würde. Planck erhielt wiederum kein Honorar. ${ }^{47}$

Die Autoren, die für C.H.Beck schrieben, stammten vor allem aus dem süddeutschen Raum, mehrheitlich aus Bayern, aber auch aus Württemberg und Baden. Sie gehörten fast ausschließlich dem protestantischen und bildungsbürgerlichen Milieu an. Wie ihr Publikum repräsentierten sie die liberale und konservative Öffentlichkeit; katholische Autoren waren selten, sozialdemokratische wurden überhaupt nicht verlegt. Hauptberufliche Schriftsteller fanden nicht nach Nördlingen, denn C.H.Beck war kein Verlag für schöne Literatur, auch wenn diese Sparte nicht völlig ausgeklammert wurde. Entsprechende Anfragen leitete Rohmer in der Regel weiter, ${ }^{48}$ es sei denn, es bestanden persönliche Verbindungen zu dem Autor oder verlegerische Interessen. An dem harten Wettbewerb um vielversprechende Manuskripte im belletristischen Literaturbetrieb beteiligte sich Rohmer folglich in aller Regel nicht. Seine Autoren waren vielmehr Universitätsprofessoren, Gymnasiallehrer, Pfarrer, Juristen und Verwaltungsbeamte, die im Nebenerwerb schrieben und denen keine exorbitanten Honorare bezahlt werden mussten. ${ }^{49}$ 
Übersetzungen ausländischer Werke bildeten keinen festen Bestandteil des Programms, sondern waren Gelegenheitsprodukte, die nicht zuletzt politische und weltanschauliche Vorlieben des Verlegers spiegelten. So brachte Ernst Rohmer 1875 die deutsche Übersetzung einer Broschüre über «Protestantismus und Katholizismus in ihren Beziehungen zur Freiheit und Wohlfahrt der Völker» heraus, die der belgische Nationalökonom Émile de Laveleye aus Liège verfasst und zu der Johann Caspar Bluntschli eine Vorrede geschrieben hatte. Die Schrift, die William E. Gladstone ins Englische übertragen hatte, schien Rohmer interessant, da sie ihm im Kulturkampf «ein immerhin bedeutungsvolles Moment anzuregen» schien. Für das deutsche Publikum seien «die Bemerkungen über die wirtschaftliche Superiorität der protestantischen Bevölkerungen, wenn auch nicht ganz neu, so doch um so einleuchtender». ${ }^{50}$

Eine Herausforderung, die Ernst Rohmer zu bestehen hatte, war die Anpassung an den entstehenden literarischen Massenmarkt. So investierte er in auflagenstarke, populäre Schriften. Zwischen 1871 und 1882 erschienen 21 Bände der Reihe «Deutsche Volks- und Jugendschriften», die zwischen 6o Pfennig und einer Mark kosteten. ${ }^{51}$ Die Mehrzahl der Heftchen war zwischen 80 und 180 Seiten stark, gab sich deutschnational, griff auf die patriotische Geschichtsschreibung zurück und idealisierte das Soldatenleben. Krieg wurde indes nicht realistisch, sondern «idyllisch verbrämt», wie auch ein Blick in Karl Geyers «Erlebnisse eines württembergischen Feldsoldaten im Kriege gegen Frankreich und im Lazarett zu Paris 1870/71» zeigte, die allerdings erst 1890 erschienen. Repräsentativ für das literarische Niveau dieses Genus dürfte die folgende Passage sein: «Die einen galoppierten auf Beutepferden durch die Gassen des Dorfes. Andere saßen, behaglich ihre Pfeife rauchend, in bunten Gruppen vor den Häusern; einer schoss eben als wir vorbeimarschierten, einer ahnungslos im Freien sich ergehenden Kuh mit seinem Zündnadelgewehr durch die Stirne, dass sie brüllend zusammenbrach. ${ }^{52} 1910$ ging das Buch in den Verlag von W. Kohlhammer in Stuttgart über.

Mitunter zahlte sich Rohmers verlegerische Hartnäckigkeit aus. So warb er lange um Friedrich Theodor Vischer, bis der berühmte Philosoph aus der Tübinger Schule und bekannte Satiriker, der sogar Goethes «Faust» persifliert hatte, C.H.Beck sein Epos «Der deutsche Krieg von 1870-71» anbot, das 1873 als «ein Heldengedicht aus dem Nachlass des seligen Philipp Ulrich Schartenmayer» publiziert wurde. Mancher Vers soll in Rohmers Haus in Nördlingen geschmiedet worden sein. Der Verleger sah es als sein Verdienst an, dass der alte «Stockpartikularist», der in der Paulskirche als 
Linksdemokrat agitiert hatte, «mit seinen politischen Sympathien im großdeutschen Lager» gestanden und «auch noch nach dem Jahre 1866 ein erklärter Gegner der Politik Preußens und Bismarcks» gewesen war, ${ }^{53}$ sich nach dem Deutsch-Französischen Krieg zur kleindeutschen Partei bekehrte und der Reichseinigung von oben Beifall spendete. ${ }^{54}$ Vischer schlug in den vier Gesängen seines Heldenepos nationalistische Töne an. Der militärische Erfolg über Frankreich und die deutsche Einheit versöhnten ihn mit dem Eisernen Kanzler: «In Versailles stolzen Räumen, / Die sich’s wohl nicht ließen träumen, / Die einst andre Zeit gesehn, / Was ist da indes geschehn? [...] Deutschland hat nicht nur den Frieden, / Noch viel mehr ist ihm beschieden: / Dass der Herde nun ein Hirt / Endlich doch bescheret wird.» ${ }^{55}$ Allerdings blieb die Angst vor der preußischen Hegemonie: «Doch verzeih es mir, Herr Preuße: / Sei dabei nicht allzuweise! / Greif nicht, wo es nicht muss sein, / Mit zu scharfem Messer ein! [...] / Sagt statt Deutsch nicht immer Preußisch / Dies erscheint uns fast karthäusisch / Wie wenn einer für die Welt / Seinen Kloster-Orden hält.» ${ }^{56}$ Am Ende bekannte der einstige Linke seine Loyalität zu Kaiser und Reich: «Er ist kein so Ungeheuer, / Ist und bleibt des Reichs getreuer / Ordentlicher Bürgersmann.» ${ }^{57}$ Solche Sätze schrieben sich für einen ehemals großdeutsch gesinnten Liberalen in der Tat besser unter Pseudonym. Das Büchlein erschien, dank Becks Bemühungen, rechtzeitig vor Weihnachten und wurde sofort zu einem Bestseller. «Schartenmayer's Volks-Epos hat zunächst in Schwaben einen glänzenden Erfolg gemacht. Heute ist die zweite Auflage erschienen. Von beiden Auflagen sind schon gegen 4,00o Exemplare verkauft», schrieb Rohmer Ende 1873 an Bluntschli. ${ }^{58} \mathrm{Zu}$ Recht hoffte Rohmer auf viele Auflagen: Die dritte und vierte Auflage erschienen 1874, die fünfte 1878 und die sechste 1904. Durch dieses Buch wurde der Verlag auf einen Schlag im neu entstandenen Reich bekannt. C.H.Beck hätte nicht besser in den Literaturbetrieb des Kaiserreichs starten können.

Der Briefwechsel mit Vischer umreißt zugleich die Grenzen des Einflusses, den persönliche Verbindungen auf die verlegerische Entscheidung, ein Buch zu veröffentlichen, nehmen konnten: Vischer versuchte Anfang 1878 , das Buch, das sein Sohn Robert über Luca Signorelli und die italienische Renaissance geschrieben hatte, C.H.Beck anzudienen. Drei kostspielige Reisen nach Italien habe sein Sohn unternommen, ließ er Rohmer wissen, schwitzend Werke, Urkunden und Chroniken studiert und drei Jahre gearbeitet, und jetzt finde sich kein Verleger. Die Situation bot ihm Anlass, grundsätzliche Überlegungen über den wissenschaftlichen Buchmarkt anzustellen: «Es gehört zum Schicksal der Gelehrtenlaufbahn, für so viel 
Schweiß nicht nur nichts zu erwerben, sondern noch froh sein zu müssen, wenn die Frucht nur in die Welt treten kann.» ${ }^{59}$ Vischer hatte gehofft, dass das kunstgeschichtliche Werk seines Sohnes in Nördlingen gedruckt würde. Doch vergeblich: Rohmer winkte ab, da er augenscheinlich die hohen Kosten scheute. Das Buch des angehenden Kunsthistorikers erschien 1879 bei Veit in Leipzig.

\section{Verlagsalltag}

Die Korrespondenz mit den Autoren belegt, dass Ernst Rohmer systematisch für die von ihm verlegten Bücher warb, sich um Rezensionen bemühte, Inserate in eigenen und fremden Schriften schaltete, Ansichtsexemplare versandte und Besprechungen akribisch sammelte, die er den Autoren mitteilte. Diese mussten dem Verlag auch Adressen von Multiplikatoren nennen. ${ }^{60}$ Bluntschlis «Staatslehre für Gebildete» versandte er 1874, an über vierzig Zeitungen; zugleich wurde die Neuerscheinung bis Weihnachten offensiv beworben. ${ }^{61}$ Seine Autoren waren zugleich die wichtigsten Ratgeber für neue Projekte. Nach der Reichsgründung dachte Rohmer daran, «ein Reichslexikon, eine Encyklopädie des öffentlichen Rechts, des Organismus und der wichtigsten statistischen Verfahren des deutschen Reiches» zu initiieren. Er fragte bei Bluntschli an, der aber offenbar nicht interessiert war. ${ }^{62}$

Wie viel Mühe Rohmer darauf verwandte, seine Bücher und damit auch seinen Verlag durch positive Besprechungen in der Öffentlichkeit bekannt zu machen, erhellen seine Bemühungen um ein schwaches Werk seines jungen Autors Adolf Wilbrandt. Der hatte 1863 mit gerade einmal 26 Jahren durch Karl Braters Vermittlung bei C.H.Beck eine Kleistbiographie veröffentlicht und verlegte in Nördlingen im Folgejahr seinen ersten Roman, «Geister und Menschen».63 Rohmer, der den jungen Journalisten der «Münchner Neuesten Nachrichten» persönlich schätzte, war von dem dreibändigen Werk nicht begeistert, wie er Bluntschli schrieb: Der Roman habe «weder Brater noch mich befriedigt, so manches Schöne auch drin ist, und wir sind sehr begierig, welches Gebiet er als das seine wohl noch erkämpfen wird». ${ }^{64}$ Später wurde er noch deutlicher: «Wilbrandt's Roman ist freilich sehr verunglückt.» In der Ausführung sei er «ganz verfehlt».65 Damit entsprach seine Einschätzung dem späteren Urteil des Autors, der sein Jugendwerk als «ein wundervoll missratenes Buch» charakterisierte. ${ }^{66}$ Dennoch wollte Rohmer seinerzeit das literarische Debüt seines Autors 
auch nicht durch die Kritik vernichtet sehen, sondern versuchte, Friedrich Theodor Vischer zu überreden, den Band zu besprechen - «freilich mit Nachsicht». Der Verfasser, ein «jüngerer Freund», gehöre «zu den an Charakter trefflichsten, an Geist begabtesten Menschen», denen er in seinem Leben begegnet sei. «Hat der Roman auch viele Fehler und ist möglicherweise die Poesie gar nicht das Gebiet meines Freundes, so wäre es mir gerade wegen seiner künftigen Entwicklung ein großes Anliegen, dass das Buch mit Liebe für den Autor besprochen würde.» Sicherheitshalber setzte er hinzu: «Sie werden nicht missverstehen, wie ich dies meine, und dass ich nicht eine Unterdrückung berechtigter Kritik will.» ${ }^{67}$ Als Rohmer wenig später erfuhr, dass in der «Allgemeinen Zeitung» eine Besprechung beabsichtigt sei, wandte er sich nochmals an Vischer, um ihn zu bitten, diese Rezension zu übernehmen. Er täte dies nicht im eigenen Interesse, sondern «Wilbrandts wegen», «der zur Zeit als Rekonvaleszent von einer schweren Nervenaufregung in Rom» weile. Zuvor bereits hatte er mit Paul Heyse, Wilbrandts «engstem Freund», darüber gesprochen, der ihn darin bestärkt hatte, sich mit dieser Bitte an Vischer zu wenden. Im eigenen Interesse bat Rohmer darum, dass in einer allfälligen Besprechung «das Lob wenigstens nicht minder ausführlich werden möchte als der Tadel!» Er fügte an: «Auf alle Fälle aber bitte ich Sie, meinen Wunsch nicht als ein Drängen aufzufassen und die Besprechung jedenfalls zu unterlassen, wenn sie gegen Ihr Gefühl geht.» ${ }^{68}$ Damit war die Alternative deutlich: Entweder sollte eine positive Besprechung des Romans erscheinen oder gar keine. Anderthalb Jahre später versuchte Rohmer nochmals sein Glück und diente Vischer Wilbrandts Übersetzungen antiker Tragödien an, die eine antiklassizistische, «radikale Verlebendigungsstrategie verfolgten», welche ganz auf die Bühne zugeschnitten war. ${ }^{69}$ Auch diesem Werk wurde nur «spröde» Aufnahme zuteil. Der literarische Ruhm ließ sich mithin nicht herbeizwingen, schon gar nicht auf dem Feld der Belletristik, das C.H.Beck kaum bestellte. Die Wege von Rohmer und Wilbrandt trennten sich. Der Schriftsteller verlegte künftig seine Werke bei Wilhelm Hertz in Berlin. ${ }^{70}$

Auf der anderen Seite zögerte Rohmer nicht, ein Projekt zu stoppen, wenn es die Umstände verlangten. Als Friedrich Theodor Vischer ihm 1863 eine «politische Broschüre» anbot, die im Vorfeld des Frankfurter Fürstentags, zu dem der österreichische Kaiser geladen hatte, Stimmung machen und «eine vernünftige großdeutsche Auffassung» vertreten wollte, schlug Rohmer sofort ein. Er verlege die Schrift «mit größtem Vergnügen», schrieb er am 10. August 1863, «nicht nur weil sie von einem so warmen 
tüchtigen Patrioten kommt und obgleich ich zu dem nicht verrannten Teile der kleindeutschen Partei gehöre, sondern weil ich mindestens hoffe, dass Ihre so warmen ehrlichen Worte zur Klärung der Situation beitragen werden». Das Manuskript ging rasch in den Satz, 1500 Exemplare sollten gedruckt werden. Dann überschlugen sich die Ereignisse: Bismarck hinderte den preußischen König, an der Konferenz, die am 17. August begann, teilzunehmen. In Frankfurt folgte die Mehrheit der Mittelstaaten den großdeutschen Interessen Österreichs. Preußen verlangte, dass die Bundesreform durch ein Parlament durchgesetzt werden müsse. Diese strategische Intervention sollte das österreichische Projekt zu Fall bringen; zugleich war sie gegen eine zentrale Forderung der Liberalen gerichtet. Bismarck gab vor, im Interesse der Nation die liberale Verfassungstheorie gegen die Fürsten durchzusetzen. Rohmer frohlockte: Der «Fürstenkongress» habe «der nationalen Sache einen ungeheuren Impuls» gegeben. Vischers Broschüre könne hingegen nur noch «als Kritik der Vergangenheit Eindruck machen». Hektisch wurden Briefe zwischen dem Verleger und seinem Autor gewechselt. Rohmer sistierte den Druck und bat Vischer, die «gänzlich veränderten Verhältnisse» zu berücksichtigen. Die großdeutschen Argumente gegen Preußens Hegemonialanspruch, die Vischer vorgebracht hatte, würden «ihre Wirkung verfehlen». Ohnehin müsse sich jetzt die großdeutsche Partei bewegen. Er sehe es, obwohl bereits ein halber Bogen gedruckt und der ganze Satz vollendet sei, als «ein patriotisches Opfer», «die Sache einfach zu kassieren». ${ }^{71}$ Ernst Rohmer trat als Zensor auf und machte mit der Druckerpresse Politik. Der Dissens zwischen Verleger und Autor antizipierte die politische Spaltung des deutschen Liberalismus in den folgenden drei Jahren.

Rohmer ging so weit, auch in Manuskripte einzugreifen, wenn ihm Korrekturen inhaltlich oder politisch geboten schienen. Als ihm der Journalist Hermann Michael Richter nach der Reichsgründung im Sommer 1871 seine Ausführungen über «Die leitenden Ideen und der Fortschritt in Deutschland von 186o bis 1870" vorlegte, war Rohmer sehr angetan, da der Autor den Gottesbegriff Friedrich Rohmers erwähnte. Doch dann entwickelte sich «eine zeitraubende Korrespondenz», weil «die politischen Ansichten des Verfassers offenbar gefärbt sind von einem Hauche idealen großdeutschen Demokratentum's». Am Ende war Richter «halb bekehrt» und machte nach Rohmers Vorgaben «kleine Änderungen und Abstriche». Das Buch wurde überarbeitet und erschien 1873 bei C.H.Beck. ${ }^{72}$

$\mathrm{Zu}$ allen Zeiten schwierig gestaltete sich der Umgang mit Autoren, die ihre Honorare hochzutreiben oder verschiedene Verleger gegeneinander 
auszuspielen versuchten - nicht anders bei Rohmer. Zu seinen Problemfällen zählte der Kulturhistoriker und Schriftsteller Wilhelm Heinrich Riehl, der 1857 seine Schrift «Über die Pfälzer» C.H.Beck anbot, in der er «eine psychologische Charakteristik einer deutschen Volksgruppe» zeichnen wollte. ${ }^{73}$ Rohmer unterbreitete im Mai ein Angebot, und Riehl versprach ihm, dass er keinen Vertrag abschließen werde, ohne Rohmer das letzte Wort zu lassen. Doch Riehl ließ Rohmer warten. Im September schrieb er, so Rohmer, «unter vielen Flausen und mit offen liegendem schlechten Gewissen, dass er den Vertrag mit der Cotta'schen Buchhandlung abgeschlossen habe». Riehl hatte sich für seinen Hausverlag entschieden, der auch sein opus magnum, «Die Naturgeschichte des Volkes als Grundlage einer deutschen Sozialpolitik» herausbrachte. Rohmer klagte seinem Freund Bluntschli über Riehls «erbärmliches Verhalten», betrachtete wenig später die Episode dann aber als «einen Fingerzeig», seine «Kraft» nicht auf «diese dritte-Standesliteratur» zu verwenden. Und er setzte hinzu: «Ohne Zweifel hat er mich bis jetzt nur benutzt, um Cotta noch höher zu schrauben; wie er Cotta's Offerte gegen mich zu einer Erhöhung des ursprünglich von ihm selbst angenommenen Honorars benützte.» ${ }^{74}$

Ernst Rohmer führte den Verlag in einer Zeit, in der der Buchhandel mit sehr viel gravierenderen Problemen als wankelmütigen Autoren zu kämpfen hatte: Erst 1879 wurden wieder so viele Neuerscheinungen verzeichnet wie 1843. Der Produktionsanstieg blieb über Jahrzehnte bescheiden, und die Auflagen waren niedrig. Bei C.H.Beck erreichte die Zahl der Neuerscheinungen in den Repressionsjahren zwischen $185^{1}$ und 1859 nicht mehr den Stand der 184,oer Jahre. Häufig wurden nur um die 1000 Exemplare gedruckt. Gleichzeitig nahmen im deutschsprachigen Raum die Sortimentsbuchhandlungen zu, die teilweise - wie C.H.Beck - über einen eigenen Verlag, über Antiquariat und Leihbibliothek verfügten; von 1843 bis 1880 kletterte die Zahl von 887 auf 3375. Davon befanden sich gut 5o Prozent in Preußen, während das Königreich Bayern einen Anteil von etwa 10 Prozent aufwies. ${ }^{75}$ Der Buchmarkt stagnierte zwar nicht, aber er wuchs nur bescheiden und war krisenanfällig.

Doch dem Verlag in Nördlingen ging es nicht schlecht. 1856 konnte das Verlagsgebäude durch den Ankauf eines benachbarten Anwesens vergröBert werden, in das zunächst das Antiquariat einzog. ${ }^{76}$ Rohmer selbst sprach damals schon von dem «großen Geschäft», dessen Umzug mühsam sei. ${ }^{77}$ Statt in neue Titel investierte man in den Antiquariatsbuchhandel. Der Handel mit Büchern aus säkularisierten Kloster- und mediatisierten Adelsbibliotheken schien sich zu lohnen, obschon das Überangebot an 
alten Drucken die Preise drückte. ${ }^{78}$ Man bediente zahlungskräftige institutionelle Kunden wie die Königliche Staats- und Hofbibliothek in München, die von Karl Halm, Ernst Rohmers ehemaligem Lehrer, geleitet wurde. Der Klassische Philologe füllte die Kasse der Bibliothek durch umstrittene Verkäufe und verwandte die Erlöse zum Ankauf wertvoller Bestände. C.H.Beck bot der Bibliothek Sammlungen und Autographen zum Kauf an. Dabei kam es jedoch bisweilen zum Dissens über den Preis, und auch der Vorwurf der «Unbilligkeit», d. h. überzogener Forderungen, stand mehr als einmal im Raum und zwang das Antiquariat zum Entgegenkommen, um den wichtigen Kunden nicht zu verlieren. ${ }^{79}$

1863 arbeiteten 38 Personen für C.H.Beck, davon 26 in der Buch- und Steindruckerei und zwölf im Sortiment. ${ }^{80} 1865$ wurde eine neue Schnellpresse des bekannten Herstellers Koenig und Bauer aus Oberzell bei Würzburg angeschafft, die allerdings im April stillstand, weil auch in Nördlingen die Drucker streikten. ${ }^{81}$ Die Bindung der Angestellten an das Unternehmen war jedoch eng; die meisten kamen aus Nördlingen, und viele blieben dem Betrieb über Jahrzehnte treu. ${ }^{82}$ Man war gerüstet für den Aufschwung in den Jahren nach 1866. 1875 bezog die Druckerei ein neues, teures Gebäude. Doch die Depression im Gefolge der ersten Weltwirtschaftskrise von 1873 machte auch C.H.Beck zu schaffen. 1875 klagte Rohmer, im Verlagsbuchhandel niemals schlechtere Zeiten erlebt zu haben. Es gingen «fast nur Schulbücher» und «seltsamer Weise Prachtwerke». Die wirtschaftliche Krise drücke auf den Mittelstand, und die niedrige Besoldung der Geistlichen und Beamten wirke sich «unendlich nachteilig auf den Buchhandel» aus. ${ }^{87}$ Noch 1877 brachte «der furchtbar schlechte Geschäftsgang» C.H.Beck «starke Verluste». ${ }^{84}$

\section{Im Kaiserreich angekommen}

Dennoch standen die Zeichen nach der Reichsgründung auf Expansion. Zu keinem Zeitpunkt war die Existenz der C.H.Beck'schen Verlagsbuchhandlung bedroht. Manche Klage des Verlegers fiel vielleicht auch deshalb etwas lauter aus, um allzu hohe Erwartungen der Autoren zu dämpfen. Von gesetzlichen Regelungen auf Reichsebene wie zur Gewerbefreiheit (1871), zum Urheberschutz (1871) und zur Pressefreiheit (1874), aber auch von technischen Neuerungen in der Papierherstellung, Setzerei und Binderei profitierten Buchproduktion und Buchhandel merklich. ${ }^{85}$ C.H.Beck operierte unter Ernst Rohmer weiterhin erfolgreich als protestantischer 
Verlag, der in theologicis profiliert war, bediente den Zeitungs- und Zeitschriftenmarkt und hatte weiterhin allerlei Ratgeberliteratur und Schulbücher im Programm. Vor allem aber konnte sich C.H.Beck, wie bereits erwähnt, als juristischer Verlag etablieren, der inzwischen auf dem Gebiet des bayerischen Rechtes eine Monopolstellung innehatte und auf dem Gebiet des reichsdeutschen Rechtes und der Staatswissenschaft immerhin eine wichtige Rolle spielte. Die verbesserten Infrastrukturbedingungen erlaubten es, auch von der Provinz aus den Buchmarkt zu bedienen. Der Verwaltungsbericht der Stadt Nördlingen hält für das Jahr 1873 fest, dass in diesem Jahr 39 «Gehilfen und Arbeiter» beschäftigt wurden und sich der Umsatz auf 120 ooo Gulden belief. Zum Vergleich: Der Jahresumsatz der Rotgerberei, die ihren Hauptsitz in Nördlingen hatte, betrug 1869 230 ooo Gulden; er wurde von zwanzig Fabrikanten erzielt. ${ }^{86}$ C.H.Beck hatte als städtisches Wirtschaftsunternehmen überregionale Bedeutung. Von den Lehrlingen und Mitarbeitern, die in Nördlingen im 19. Jahrhundert tätig waren, gründeten später nicht wenige ihren eigenen Verlag: August Herzer in Würzburg, Carl Junge in Rothenburg, August Kampfhammer in Schwäbisch Gmünd, Niclaus Lehmann in Prag, Gottfried Löhe in Nürnberg, Friedrich Manz in Dillingen und Schwäbisch Gmünd, Albert Moser in Tübingen, Albert Thilo Schiefer in Nürnberg, G.H. Stifel in Traunstein, Adalbert Stufer in Würzburg und Carl Hermann Trenkle in Rothenburg. ${ }^{87}$

Der betagte Ernst Rohmer stellte wie so viele Liberale seiner Generation fest, dass er in der Politik «täglich konservativer» werde und der Liberalismus «seit der Gründung des Reiches in und außerhalb der Gesetzgebung recht nach vielen Seiten hin als echter Radikalismus sich ausgewachsen hat, wie es in den Parteien so meisterhaft gezeichnet ist». Schuld daran seien «die Juden», wie er meinte. Mit vielen liberalen Bildungs- und Wirtschaftsbürgern teilte er antijüdische Vorurteile. Als 1880 in Berlin der Antisemitismusstreit tobte, stand er nicht auf der Seite Theodor Mommsens, sondern «entschieden bei Treitschke. Es ist traurig, dass in unserm noch so wenig fest begründeten Reiche immer neue schwere Fragen auftauchen, aber der deutsche Volksgeist reagiert hier gewissermaßen aus der Tiefe heraus gegen den Christentum zersetzenden Geist der Reformjuden in der Presse und Gesetzgebung und gegen die ungeheure Überhebung des Judentums überhaupt, seit es von Jahr zu Jahr zu größerer Macht gelangt. Man sucht die Ursache oft in Neid gegenüber der größeren semitischen Begabung. Es mag ja etwas dabei sein, aber gewiss ist, dass die ungeheure Mehrheit der Christen so verwerfliche Geschäfte, 
wie sie die Juden machen, aus Prinzip nicht macht.» ${ }^{88}$ Auch in Nördlingen wurden mithin antisemitische Stereotypen hoffähig. Der politische Antisemitismus fand indes keinen Eingang in das Programm des liberal und national gesinnten Verlags.

Ernst Rohmer hatte zwei Töchter und vier Söhne, von denen einer, Emil, der an Epilepsie litt, in jungen Jahren starb. Eugen, Theodor und Gustav wuchsen heran und studierten Rechtswissenschaft und Medizin und standen nicht bereit, den Verlag zu übernehmen. So traten die beiden Stiefsöhne Carl und Oskar Beck in das Geschäft ein. Beide besuchten die Lateinschule in Nördlingen und lernten nach der Schulzeit als Lehrlinge das Buchhandelsgeschäft. Oskar Beck, der den Besuch des Bayreuther Gymnasiums wegen eines Augenleidens abbrechen musste und Mitte Mai 1868 seine Lehre in Nördlingen begann, erwarb 1872 nachträglich das Reifezeugnis am Wilhelmsgymnasium in München. Durch ein hartnäckiges Knieleiden, das er sich beim Militär zugezogen hatte, verzögerte sich sein Eintritt in das Geschäft bis $1874 .{ }^{89}$ Rohmer ließ nie einen Zweifel daran, dass sein Stiefsohn Oskar das begabteste unter seinen Kindern war. Er war ihm «eine große Stütze im Geschäft», und er lobte sein «großes geistiges und ideales Interesse bei einer feinen und tüchtigen Bildung» und «sein bedeutendes praktisches Geschick».90 Schließlich wurde Oskar Beck am 1. Januar 1878 gemeinsam mit seinem älteren Bruder Carl Teilhaber der Firma. ${ }^{91}$ Nachdem sein Onkel Wilhelm Beck, der Rohmer über Jahre als Freund treu und selbstlos unterstützt hatte, ${ }^{92}$ am 12. Juni 1879 gestorben war, übernahm Oskar das Rechnungswesen. Am 1. Juli 1884, zog sich Ernst Rohmer ganz aus dem Geschäft zurück. Es ging an diesem Tag mit allen seinen Zweigen - Verlag, Buchdruckerei, «Nördlinger Anzeigeblatt», Sortiment, Antiquariat - an Oskar Beck über. Carl Beck, so hält die Familienüberlieferung fest, fühlte sich «der Verantwortung in der Geschäftsführung» nicht gewachsen. ${ }^{97}$ Ernst Rohmer starb am 23. August 1897 in seinem 78 . Lebensjahr. 
https://doi.org/10.17104/9783406654015-169, am 26.04.2023, 17:31:47

Open Access - (c) EY EY - http://www.beck-elibrary.de/agb 